\title{
Robust Measurement Matrix Design Based on Compressed Sensing for DOA Estimation
}

\author{
Zhikai HUANG, Wei WANG \\ College of Automation, Harbin Engineering University, Nantong Street 145, Harbin, 150001, China \\ xiaokai407@hrbeu.edu.cn,wangwei407@hrbeu.edu.cn \\ Submitted September 20, 2018 / Accepted January 1, 2019
}

\begin{abstract}
It has been well known that Massive multipleinput-multiple-output (MIMO) radar can provide an excellent performance in direction of arrival (DOA) estimation. However, the significant increasing data size will seriously reduce the computational efficiency in practical application. Although compressed measurement can reduce data size and computational complexities, improper compression will enhance the environment noise. In this paper, a robust measurement matrix is designed to reduce data size and environment noise. Different from the general compressed sensing (CS) schemes, the optimization function is established by considering the overall mutual coherence of dictionary and the energy of measurement matrix, which is more suitable for noisy environment. The optimization function is highly non-convex due to the rank shrinkage of measurement matrix. To solve this problem, an alternating minimization scheme based on matrix factorization and Principal Component Analysis (PCA) is proposed. Moreover, the structure of measurement matrix is designed for massive MIMO receiver. Furthermore, numerous results demonstrate this scheme has a better estimation performance than random measurement method and general CS schemes in the noisy environment.
\end{abstract}

\section{Keywords}

compressed sensing, robust measurement design, DOA estimation, sparse representation, massive MIMO

\section{Introduction}

Direction of arrival (DOA) estimation is an important research topic of array signal processing [1]. Currently, massive multiple-input-multiple-output (MIMO) radar has been widely concerned because of its benefits, such as large aperture, high degrees of freedom (DOF) and super resolution [2-4]. Although massive MIMO has great advantages in DOA estimation, its application will also be limited by the increasing data size of received signal. Thus, computational complexity is necessary to be concerned due to the high dimensional matrix operations. However, it is difficult to avoid these operations from an algorithm perspective.
In order to guarantee the efficiency and effectiveness of massive MIMO system, reasonably reducing measurement dimension is a feasible way.

Changing array structure is one of the most common ways to reduce measurement dimension. Compared with uniform linear arrays (ULA), the sparse linear arrays can achieve the equivalent performance using fewer physical antennas [5-7]. The nested array [8], [9] and the co-prime array [10] are the two most famous structures. However, it's hard to reconfigure the array structure of an existent massive MIMO. In order to reconfigure, the compressed measurement matrix is designed in hardware to replace changing exist structure [11]. In addition, compressed sensing (CS) technique provides a new perspective for selecting the optimal measurement matrix.

According to CS theory, the low mutual coherence between sparse dictionaries will provide a well recovery performance. Generally, the random matrices such as Gaussian or Bernoulli distributions which have excellent incoherence property can be adopted as measurement matrix [12]. A random measurement matrix based on Gaussian distribution is designed in [13]. However, the average mutual coherence of random matrices are not the lowest. In [14], an alternating minimization algorithm is proposed to optimize measurement matrix, which can reduce the average mutual coherence between equivalent sparse dictionaries and provide a better recovery performance than random matrices. The equiangular tight frame (ETF) criterion is used to design the measurement matrix in [15-18], which has lower average mutual coherence than [14]. Furthermore, the closed-form solution of above optimization problem is given in [18], which can avoid a heavy searching. However, these schemes are designed based on a noiseless model, which will result in a bad estimation performance at low SNR. There are some studies about measurement matrix design for enhancing system robustness [19-21]. A measurement matrix of compressed array is designed in [19] based on Cramer-Rao bound (CRB) with the prior information of received signals. Although the estimation performance of this method approaches to CRB, it is not suitable for unknown noise. In [20], measurement matrix is optimized by maximizing the mutual information of the signal, which can enhance the robustness of DOA 
estimators. However, it also need to know the distributions of targets and the SNR of environment. In [21], measurement matrix is designed by taking sparse representation error into consideration. Although this optimization scheme can suppress the noise without any prior information, a heavy linear searching steps are performed during each iteration of alternating method.

In this paper, we propose a novel approach to design the robust measurement matrix which can reduce the computational complexity of DOA estimators in noisy environment. Different from the traditional measurement matrices [13-18], we take both mutual coherence of dictionary and the energy of measurement matrix into consideration which can provide an excellent performance in the case of low SNR. Moreover, this algorithm does not require any prior information of targets and environment. Compared with [21], the measurement matrix can be figured out by matrix factorization method and Principle Component Analysis (PCA), which can avoid a heavy linear searching. Numerical results demonstrate the superiority of proposed scheme.

The remainder is arranged as follows. The spatial sparse signal model and the measurement model of DOA estimation system are established in Sec. 2. The optimization criterion and the specific optimization procedures of proposed algorithm are presented in Sec. 3. Simulation results are used to prove the validity of proposed scheme in Sec. 4. The Section 5 conclude the whole paper.

Notations: Boldface upper-case letters, boldface lowercase letters and non-boldface letters respectively denote matrices, vectors and scalars. $\mathbb{C}$ denotes the complex number field. $\mathbf{I}_{n}$ denotes a $n \times n$ identity matrix, $\mathbf{0}_{m \times n}$ denotes an allzero matrix. $(\cdot)^{-1},(\cdot)^{\mathrm{T}}$ and $(\cdot)^{\mathrm{H}}$ represent inverse, transpose, conjugate transpose operators of matrix or vector. $\operatorname{Tr}(\cdot)$ denotes the trace of matrix, respectively. $\|\cdot\|_{F}$ denotes the Frobenius norm of a matrix.

\section{System Model}

In this section, the spatial sparse signal model and the measurement model are established.

\subsection{Signal Model}

Consider that there are $P$ far-field narrow-band uncorrelated signals impinging on a $N$-element uniform linear array (ULA) from directions $\theta=\left[\theta_{1}, \theta_{2} \cdots, \theta_{P}\right]$. The array received signals $\mathbf{x}(t)$ can be described as

$$
\mathbf{x}(t)=\sum_{p=1}^{P} \mathbf{a}\left(\theta_{p}\right) s_{p}(t)+\mathbf{n}(t)=\mathbf{A}(\theta) \mathbf{s}(t)+\mathbf{n}(t)
$$

where $\mathbf{A}(\theta)=\left[\mathbf{a}\left(\theta_{1}\right), \mathbf{a}\left(\theta_{2}\right), \cdots, \mathbf{a}\left(\theta_{\mathrm{P}}\right)\right] \in \mathbb{C}^{\mathrm{N} \times \mathrm{P}}$ is the array manifold, $\mathbf{a}\left(\theta_{p}\right)=\left[1, \mathrm{e}^{\frac{-\mathrm{j} 2 \pi d \sin \left(\theta_{p}\right)}{\lambda}}, \cdots, \mathrm{e}^{\frac{-\mathrm{j} 2 \pi(N-1) d \sin \left(\theta_{p}\right)}{\lambda}}\right]^{\mathrm{T}}$ is the steering vector corresponding to the $p$-th signal, $\lambda$ denotes the carrier wavelength, $d$ represents the distance between adjacent elements, $\mathbf{s}(t)=\left[\mathrm{s}_{1}(\mathrm{t}), \mathrm{s}_{2}(\mathrm{t}), \cdots, \mathrm{sp}_{\mathrm{P}}(\mathrm{t})\right]^{\mathrm{T}} \in \mathbb{C}^{\mathrm{P} \times 1}$ denotes the signal vector, $\mathbf{n}(t) \in \mathbb{C}^{N \times 1}$ denotes the noise matrix.
If we divide the spatial domain into $L(P \ll L)$ grids, the sampling signal of all grids can be considered as the sparse signal. Then the sparse signal model of DOA can be expressed as

$$
\mathbf{x}(t)=\mathbf{D S}(t)+\mathbf{n}(t)
$$

where $\mathbf{D}=\left[\mathbf{a}\left(\theta_{1}\right), \mathbf{a}\left(\theta_{2}\right), \cdots, \mathbf{a}\left(\theta_{L}\right)\right] \in \mathbb{C}^{N \times L}$ is the sparse dictionary, $\mathbf{S}(t) \in \mathbb{C}^{L \times 1}$ denotes the spatial sparse signal vector.

\subsection{Measurement Model}

The above model is established under the condition of a dedicated radio frequency $(\mathrm{RF})$ receiver chain for each antenna element. To reduce the data size and the number of RF receiver chains, a measurement matrix $\boldsymbol{\Phi}$ can be designed in the masssive MIMO receiver. The measurement matrix is a $M \times N,(M<N)$ complex matrix, which can be expressed as

$$
\boldsymbol{\Phi}=\left[\begin{array}{cccc}
W_{11} \mathrm{e}^{\mathrm{j} \omega_{11}} & W_{12} \mathrm{e}^{\mathrm{j} \omega_{12}} & \cdots & W_{1 N} \mathrm{e}^{\mathrm{j} \omega_{1 N}} \\
W_{21} \mathrm{e}^{\mathrm{j} \omega_{21}} & W_{22} \mathrm{e}^{\mathrm{j} \omega_{22}} & \cdots & W_{2 N} \mathrm{e}^{\mathrm{j} \omega_{2 N}} \\
\vdots & \vdots & \ddots & \vdots \\
W_{M 1} \mathrm{e}^{\mathrm{j} \omega_{M 1}} & W_{M 2} \mathrm{e}^{\mathrm{j} \omega_{M 2}} & \cdots & W_{M N} \mathrm{e}^{\mathrm{j} \omega_{M N}}
\end{array}\right]
$$

where $W$ and $\omega$ respectively denote the amplitude and phase. Furthermore, the amplitude and phase can be implemented by amplifier and phaser in hardware. The architecture of measurement system can be represented in Fig. 1.

It is worth noting that $\boldsymbol{\Phi}$ can compress the $N$-element array into a $M$-dimensional measurement. The compressed measurement can be expressed as

$$
\mathbf{y}(t)=\boldsymbol{\Phi} \mathbf{x}(t) \in \mathbb{C}^{M \times 1} .
$$

Additionally, the measurement compression ratio $\beta$ can be defined as

$$
\beta=\frac{N}{M}
$$

With substituting (2) into (4), the measurement model is derived as

$$
\mathbf{y}(t)=\boldsymbol{\Phi D S}(t)+\boldsymbol{\Phi} \mathbf{n}(t)
$$

where $\boldsymbol{\Phi} \in \mathbb{C}^{M \times N}$ is the measurement matrix. Obviously, the application of $\boldsymbol{\Phi}$ can extremely reduce the data size and the computational complexities of DOA estimators. However, inappropriate dimensionality reduction operation will lead to performance loss and increase noise. Thus, choosing optimal $\boldsymbol{\Phi}$ is the key operation to ensure estimation accuracy. In next section, an algorithm is proposed to design optimal measurement matrix $\boldsymbol{\Phi}$. 


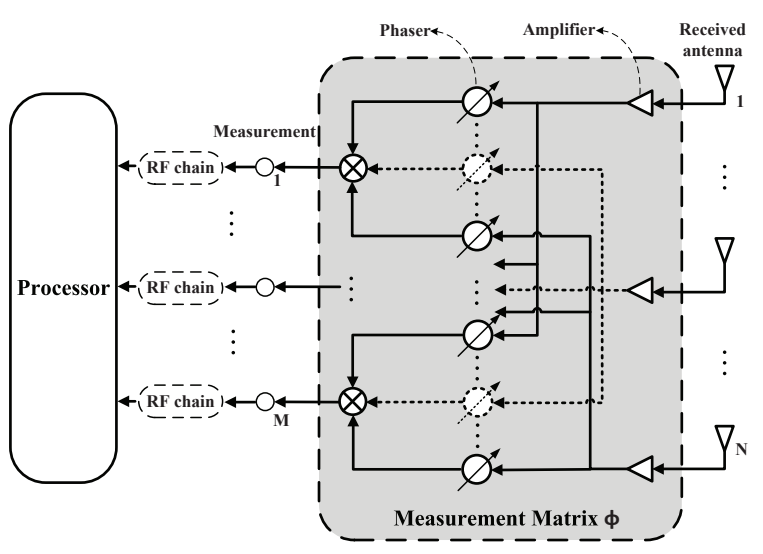

Fig. 1. The architecture of measurement system.

\section{Measurement Matrix Design Based on CS}

In this section, the optimization problem is formulated. In addition, an alternating minimization scheme based on matrix factorization and PCA method is proposed to design measurement matrix.

\subsection{The Optimization Criterion and Problem Formulation} defined as

According to CS theory, the equivalent dictionary $\mathbf{E}$ is

$$
\mathbf{E}=\boldsymbol{\Phi} \mathbf{D}
$$

where $\boldsymbol{\Phi} \in \mathbb{C}^{M \times N}(M<N)$ is the measurement matrix, D $\in \mathbb{C}^{N \times L}$ is the sparse dictionary. Then the mutual coherence of equivalent dictionary mentioned in [14] is defined as

$$
\mu(\mathbf{E}) \triangleq \max _{1 \leq i \neq j \leq M} \frac{\left|\mathbf{E}_{i}^{\mathrm{H}} \mathbf{E}_{j}\right|}{\left\|\mathbf{E}_{i}\right\|_{2}\left\|\mathbf{E}_{j}\right\|_{2}}
$$

where $\mathbf{E}_{i}$ and $\mathbf{E}_{j}$ are the $i$-th and $j$-th columns of $\mathbf{E}$, respectively. If the columns of $\mathbf{E}$ have been normalized, the Gram matrix $\mathbf{G} \triangleq \mathbf{E}^{\mathrm{H}} \mathbf{E}$ can be used to express mutual coherence.

To guarantee the recovery accuracy, the low bound of mutual coherence $\sqrt{\frac{L-M}{M(L-1)}},(M \ll L)$ is chosen as the recovery condition. The equiangular tight frame (ETF) is proposed to design measurement matrix which is satisfied the recovery condition [15], [16]. Moreover, the optimization problem with the ETF criterion can be depicted as

$$
\min _{\mathbf{\Phi}, \mathbf{H}_{\mathbf{H}}}\|\mathbf{G}-\mathbf{H}\|_{\mathrm{F}}
$$

where the $\mathbf{H} \in \mathbb{C}^{L \times L}$ is the target of Gram matrix that belongs to following domain

$$
\mathbf{H}_{\varepsilon} \triangleq\left\{\mathbf{H}\left|\mathbf{H}=\mathbf{H}^{\mathrm{H}}, \mathbf{H}(i, i)=1, \max _{i \neq j}\right| \mathbf{H}(i, j) \mid \leq \varepsilon\right\},
$$

in which $\varepsilon$ is a constant to control mutual coherence.
In practice, if $\boldsymbol{\Phi}$ is only chosen by following the ETF criterion, the noise term ( $\boldsymbol{\Phi \mathbf { n }}$ ) will be enhanced in noisy environment. To suppress the increasing noise $(\boldsymbol{\Phi n})$, the value of $\|\boldsymbol{\Phi} \mathbf{n}\|_{\mathrm{F}}^{2}$ should be taken into consideration in measurement matrix design [21]. However, it is hard to obtain the precise information of environment noise. Then the following norm property can be used to suppress $\|\boldsymbol{\Phi} \mathbf{n}\|_{\mathrm{F}}^{2}$.

$$
\|\boldsymbol{\Phi} \mathbf{n}\|_{\mathrm{F}}^{2} \leq\|\boldsymbol{\Phi}\|_{\mathrm{F}}^{2}\|\mathbf{n}\|_{\mathrm{F}}^{2} .
$$

Therefore, we can suppress the $\|\boldsymbol{\Phi} \mathbf{n}\|_{\mathrm{F}}^{2}$ by minimizing $\|\boldsymbol{\Phi}\|_{\mathrm{F}}^{2}$. Moreover, the robust optimization problem can be established as

$$
\min _{\mathbf{\Phi}, \mathbf{H}_{\mathbf{H}}}\|\mathbf{G}-\mathbf{H}\|_{\mathrm{F}}+\gamma\|\boldsymbol{\Phi}\|_{\mathrm{F}}^{2},
$$

where the $\gamma$ is a trade-off parameter.

\subsection{Optimization Design of Robust Measurement Matrix}

Different from the dictionary in traditional CS, the overcomplete sparse dictionary $\mathbf{D}$ is designed by its real array structure and the grids of spatial domain. Note that the grid number of spatial domain $(L)$ should be bigger than the number of antennas $(N)$. From the physical structure of array manifold, we notice that the sparse dictionary $\mathbf{D}$ has full row rank. With singular value decomposition (SVD), the sparse dictionary $\mathbf{D}$ can be decomposed into

$$
\mathbf{D}=\mathbf{U}\left[\begin{array}{ll}
\boldsymbol{\Lambda}_{N \times N} & \mathbf{0}_{N \times(L-N)}
\end{array}\right] \mathbf{V}^{\mathrm{H}}
$$

where $\boldsymbol{\Lambda}_{N \times N}$ is the singular value matrix of the sparse dictionary, $\mathbf{U}$ and $\mathbf{V}$ are matrices with proper dimensions. For convenience of calculation, the $\boldsymbol{\Lambda}_{N \times N}$ can be reprocessed by

$$
[\boldsymbol{\Lambda}]_{k, k}=\left\{\begin{array}{cc}
\left|[\boldsymbol{\Lambda}]_{k, k}\right|, & \text { if }\left|[\boldsymbol{\Lambda}]_{k, k}\right| \geq \delta_{\min } \\
\delta_{\min }, & \text { otherwise }
\end{array}\right.
$$

where $[\boldsymbol{\Lambda}]_{k, k}$ denotes the $k$-th $(k \leq N)$ entry of $\boldsymbol{\Lambda}_{N \times N}, \delta_{\text {min }}$ denotes a small constant. Then the sparse dictionary $\tilde{\mathbf{D}}$ can be normalized by

$$
\tilde{\mathbf{D}}=\sqrt{N} \frac{\mathbf{D}}{\|\mathbf{D}\|_{\mathrm{F}}^{2}}
$$

The Gram matrix can be calculated by

$$
\tilde{\mathbf{G}}(\boldsymbol{\Phi})=\tilde{\mathbf{D}}^{\mathrm{H}} \boldsymbol{\Phi}^{\mathrm{H}} \boldsymbol{\Phi} \tilde{\mathbf{D}} \text {. }
$$

The target Gram matrix $\mathbf{H}$ can be constructed by

$$
\mathbf{H}(i, j)=\left\{\begin{array}{cc}
1, & \text { if } i=j \\
\tilde{\mathbf{G}}(i, j), & \text { if }|\tilde{\mathbf{G}}(i, j)| \leq \varepsilon, i \neq j \\
\varepsilon \cdot \operatorname{sign}(\tilde{\mathbf{G}}(i, j)), & \text { otherwise }
\end{array}\right.
$$

where $\mathbf{H}(i, j)$ denotes the off-diagonal entry of $\mathbf{H}, \tilde{\mathbf{G}}(i, j)$ denotes the the off-diagonal entry of $\tilde{\mathbf{G}}$.

Consequently, the optimization problem (12) can be rewritten as

$$
\min _{\boldsymbol{\Phi}, \mathbf{H}}\left\|\mathbf{H}-\tilde{\mathbf{D}}^{\mathrm{H}} \boldsymbol{\Phi}^{\mathrm{H}} \boldsymbol{\Phi} \tilde{\mathbf{D}}\right\|_{\mathrm{F}}^{2}+\gamma\|\boldsymbol{\Phi}\|_{\mathrm{F}}^{2} .
$$


By defining $\mathbf{T}=\boldsymbol{\Phi}^{\mathrm{H}} \boldsymbol{\Phi}$, the problem (18) is equivalent to

$$
\begin{aligned}
& \min _{\mathbf{T}, \mathbf{H}}\left\|\mathbf{H}-\tilde{\mathbf{D}}^{\mathrm{H}} \mathbf{T} \tilde{\mathbf{D}}\right\|_{\mathrm{F}}^{2}+\gamma \operatorname{Tr}(\mathbf{T}) \\
& \text { s.t. } \operatorname{rank}(\mathbf{T}) \leq M, \mathbf{T} \geq 0 .
\end{aligned}
$$

Clearly, the above function of $\boldsymbol{\Phi}$ is highly non-convex. Thus, the linear search method cannot be directly used to achieve the optimal. To solve this problem, the non-convex problem (19) can be relaxed to

$$
\min _{\mathbf{T}, \mathbf{H}}\left\|\mathbf{H}-\tilde{\mathbf{D}}^{\mathrm{H}} \mathbf{T} \tilde{\mathbf{D}}\right\|_{\mathrm{F}}^{2}+\gamma \operatorname{Tr}(\mathbf{T}) .
$$

It is worth noting that the cost function of $\mathbf{T}$ is convex for every fixed $\mathbf{H}$. Then the gradient $\nabla_{\mathbf{T}} f(\mathbf{T}, \mathbf{H})$ and the second order gradient $\nabla_{\mathbf{T}}^{2} f(\mathbf{T}, \mathbf{H})$ of the cost function in terms of $\mathbf{T}$ can be respectively calculated as

$$
\begin{gathered}
\nabla_{\mathbf{T}} f(\mathbf{T}, \mathbf{H})=2\left(\tilde{\mathbf{D}} \tilde{\mathbf{D}}^{\mathrm{H}} \mathbf{T} \tilde{\mathbf{D}} \tilde{\mathbf{D}}^{\mathrm{H}}-\left(\tilde{\mathbf{D}} \mathbf{H} \tilde{\mathbf{D}}^{\mathrm{H}}-\frac{\gamma}{2} \mathbf{I}_{\mathrm{N}}\right)\right), \\
\nabla_{\mathbf{T}}^{2} f(\mathbf{T}, \mathbf{H})=\tilde{\mathbf{D}} \tilde{\mathbf{D}}^{\mathrm{H}} \tilde{\mathbf{D}} \tilde{\mathbf{D}}^{\mathrm{H}}>\mathbf{0} .
\end{gathered}
$$

Obviously, the optimal $\mathbf{T}$ with fixed $\mathbf{H}$ of problem (20) can be achieved under the condition $\nabla_{\mathbf{T}} f(\mathbf{T}, \mathbf{H})=\mathbf{0}$. Thus, the optimal $\mathbf{T}_{\text {opt }}^{\prime}$ can be derived as

$$
\mathbf{T}_{\mathrm{opt}}^{\prime}=\tilde{\mathbf{A}}^{-\mathrm{H}} \mathbf{Q} \tilde{\mathbf{A}}^{-1}
$$

where $\mathbf{Q}=\left(\tilde{\mathbf{D}} \mathbf{H} \tilde{\mathbf{D}}^{\mathrm{H}}-\frac{\gamma}{2} \mathbf{I}_{\mathrm{N}}\right)$ and $\tilde{\mathbf{A}}=\tilde{\mathbf{A}}^{\mathrm{H}}=\tilde{\mathbf{D}} \tilde{\mathbf{D}}^{\mathrm{H}}$. Additionally, $\tilde{\mathbf{A}}$ is a $N \times N$ full rank matrix. With the eigenvalue decomposition (EVD), $\tilde{\mathbf{A}}$ can be decomposed into

$$
\tilde{\mathbf{A}}=\mathbf{U} \Sigma^{2} \mathbf{U}^{\mathrm{H}}
$$

where $\mathbf{U}$ and $\Sigma^{2}$ denote the eigenvector matrix and eigenvalue matrix respectively. Then the target $\mathbf{T}_{\mathrm{opt}}^{\prime}$ can be rewritten as

$$
\mathbf{T}_{\mathrm{opt}}^{\prime}=\mathbf{U} \Sigma^{-2} \mathbf{J} \Sigma^{-2} \mathbf{U}^{\mathrm{H}}
$$

where $\mathbf{J}=\mathbf{U}^{\mathrm{H}} \mathbf{Q} \mathbf{U}$. It is also worth noting that $\mathbf{T}_{\mathrm{opt}}^{\prime}$ is the solution of (20), not the solution of (18). In another word, when the rank of $\mathbf{T}_{\text {opt }}^{\prime}$ is larger than $M$, the optimal $\boldsymbol{\Phi}$ can not be decomposed from $\mathbf{T}_{\mathrm{opt}}^{\prime}$. Principal Component Analysis (PCA) method is a classical dimensionality reduction method, which can approximate the original matrix by ignoring the small eigenvalues. Thus, we reconstruct the target $\mathbf{T}_{\text {opt }}^{\prime}$ by PCA method. By decomposing $\mathbf{J}=\mathbf{V}_{\mathrm{T}} \boldsymbol{\Lambda}^{2} \mathbf{V}_{\mathrm{T}}^{\mathrm{H}}$, the eigenvalue of $\mathbf{J}$ can be changed into

$$
\tilde{\boldsymbol{\Lambda}}^{2}=\left\{\begin{array}{cc}
\boldsymbol{\Lambda}^{2}, & \text { if } \operatorname{rank}\left(\boldsymbol{\Lambda}^{2}\right) \leq M \\
{\left[\begin{array}{cc}
\boldsymbol{\Lambda}_{M}^{2} & \mathbf{0} \\
\mathbf{0} & \mathbf{0}
\end{array}\right],} & \text { otherwise }
\end{array}\right.
$$

where $\boldsymbol{\Lambda}_{M}^{2}$ consists of $M$ largest eigenvalues of $\mathbf{J}$. Moreover, the new target can be derived as

$$
\mathbf{T}_{\mathrm{opt}}^{*}=\mathbf{U} \Sigma^{-2} \mathbf{V}_{T} \tilde{\mathbf{\Lambda}}^{2} \mathbf{V}_{T}^{\mathrm{H}} \Sigma^{-2} \mathbf{U}^{\mathrm{H}} .
$$

Hence, the optimization problem (18) can be converted into

$$
\min _{\boldsymbol{\Phi}}\left\|\mathbf{T}_{\mathrm{opt}}^{*}-\boldsymbol{\Phi}^{\mathrm{H}} \boldsymbol{\Phi}\right\|_{\mathrm{F}}^{2}
$$

Remark that the above optimization problem (28) can be solved by exhaustive searching method. To avoid a heavy linear searching steps, we can use a matrix factorization method to get the nearest solution $\boldsymbol{\Phi}_{\mathrm{opt}}^{\prime}$. Furthermore, when $\mathbf{H}$ and $\tilde{\mathbf{D}}$ are fixed, the closed-form solution of (28) can be derived as

$$
\boldsymbol{\Phi}_{\mathrm{opt}}^{\prime}=\mathbf{U}_{M}\left[\begin{array}{ll}
\tilde{\boldsymbol{\Lambda}}_{M \times M} & \mathbf{0}_{M \times(N-M)}
\end{array}\right] \mathbf{V}_{T}^{\mathrm{H}} \Sigma^{-2} \mathbf{U}^{\mathrm{H}}
$$

where $\mathbf{U}_{M}$ is an arbitrary unitary matrix with proper dimension. Then we use an alternating minimization algorithm to get the optimal, which was mentioned by [22], [23]. Moreover, the convergence of the alternating minimization method can be found in [22], [23]. The initial $\boldsymbol{\Phi}_{0}$ is chosen as a random matrix. Generally, $\varepsilon$ is chosen as the low bound of mutual coherence. Above all, the robust measurement matrix design algorithm is concluded as follow:

- Step 1: Select sparse dictionary $\mathbf{D}$, the control constant $\varepsilon$, thethreshold $\delta_{\min }$ and the trade-off parameter $\gamma$.

- Step 2: Initialize measurement matrix $\boldsymbol{\Phi}$ with $\boldsymbol{\Phi}_{0}$.

- Step 3: Normalize D to $\tilde{\mathbf{D}}$ with (15).

- Step 4: Calculate the Gram matrix $\mathbf{G}$ according to (16).

- Step 5: Construct target Gram matrixH based on (17).

- Step 6: Compute the optimal target $\mathbf{T}_{\mathrm{opt}}^{\prime}$ with (23).

- Step 7: Reconstruct $\mathbf{T}_{\mathrm{opt}}^{*}$ on the basis of (27).

- Step 8: Figure out the nearest solution $\boldsymbol{\Phi}_{\text {opt }}^{\prime}$ with (29).

- Step 9: Update $\boldsymbol{\Phi}$ with the nearest solution $\boldsymbol{\Phi}_{\mathrm{opt}}^{\prime}$, and then go to the Step 4 unless the measurement matrix reaches the optimal $\boldsymbol{\Phi}_{\text {opt }}$.

Remark that the optimal measurement matrix will be rewritten as (3) and fixed in hardware by phasers and amplifers. Hencely, this proposed scheme is off-line, which means the proposed scheme will radically reduce the computational complexities of DOA estimators without considering its own.

\section{Simulation Results}

In this section, some simulations are presented to verify the effectiveness of the proposed scheme. The random measurement method [13], the Elad's algorithm [14] and the LZYCB algorithm [18] are compared with the proposed scheme.

In the simulations below, MVDR algorithm is exploited to estimate DOA. The MVDR spatial spectrum estimator can be described as

$$
P_{\mathrm{MVDR}}(\theta)=\frac{1}{\mathbf{b}^{\mathrm{H}}(\theta) \mathbf{R}_{\mathbf{y y}}^{-1} \mathbf{b}(\theta)}
$$


where $\mathbf{R}_{\mathbf{y y}}=\frac{1}{T} \sum_{t=1}^{T} \mathbf{y}(t) \mathbf{y}^{\mathrm{H}}(t)$ denotes the covariance matrix of the signal, $T$ is the snapshots, $\mathbf{b}(\theta)=\boldsymbol{\Phi a}(\theta)$ is the steering vector. The following root mean square error (RMSE) is used for performance measure

$$
R M S E=\sqrt{\frac{1}{M_{t} P} \sum_{m=1}^{M_{t}} \sum_{p=1}^{P}\left(\widehat{\theta}_{m, p}-\theta_{p}\right)^{2}}
$$

where the $M_{t}$ is the number of Monte-Carlo and the $P$ is target number. Moreover, we suppose that the massive array is chosen as a 50-element ULA and the distance between adjacent elements is half of wavelength.

In the first simulation, we discuss the convergence and selection of alternating iteration number in different tradeoff parameters. The compression ratio is chosen as 5. The spatial domain grid is divided into $0.1^{\circ}$. Then the value of cost function (18) during optimization iteration is shown in the Fig. 2.

From Fig. 2, we can notice that the proposed scheme has the same convergence rate in different $\gamma$. Moreover, when the iteration number is larger than $50, \Phi$ can be considered as approaching optimal. Thus, the iteration number can be properly chosen as 50 .

In the second simulation, we discuss the effect of compression ratio with different SNR. We assume that there is one target. The searching step is $0.01^{\circ}$. The number of snapshots is fixed to $T=100$. In each case, 5,000 Monte-Carlo are carried out. Finally, the effect of compression ratio in different SNR are shown in Fig. 3.

Figure 3 demonstrates that the estimation accuracy will decrease with the increasing compression ratio. Thus, it is important to choose a proper compression rate following different needs. To trade off the performance and data size, the compression ratio is chosen as 5 in the following simulations.

In the third simulation, we discuss the selection of tradeoff parameter $\gamma$ in different SNR. Other conditions are same as the second simulation.

According to Fig. 4, the trade-off parameter $\gamma=1$ is optimal when the range of SNR is $-15 \mathrm{~dB}$ to $-5 \mathrm{~dB}$. However, Figure 5 shows that the DOA performance has less related to $\gamma$ in the high SNR. Thus, it is proved that the constraint $\|\boldsymbol{\Phi}\|_{\mathrm{F}}^{2}$ has less effect on the DOA estimation at high SNR. To improve the robustness in noisy environment, the $\gamma$ is chosen as 1 in the following simulations.

In the fourth simulation, different measurement schemes are compared under multi-target conditions. In Fig. 6 and Fig. 7, we assume that there are 9 signals impinging from directions $\theta=\left[-8^{\circ},-6^{\circ},-4^{\circ},-2^{\circ}, 0^{\circ}, 2^{\circ}, 4^{\circ}, 6^{\circ}, 8^{\circ}\right]$, the SNR is set as $20 \mathrm{~dB}$. The other conditions are followed by the second simulation.

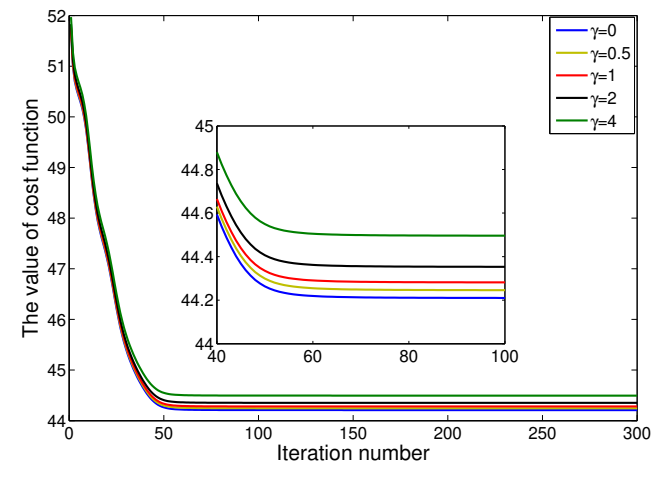

Fig. 2. The value of cost function versus iteration number.

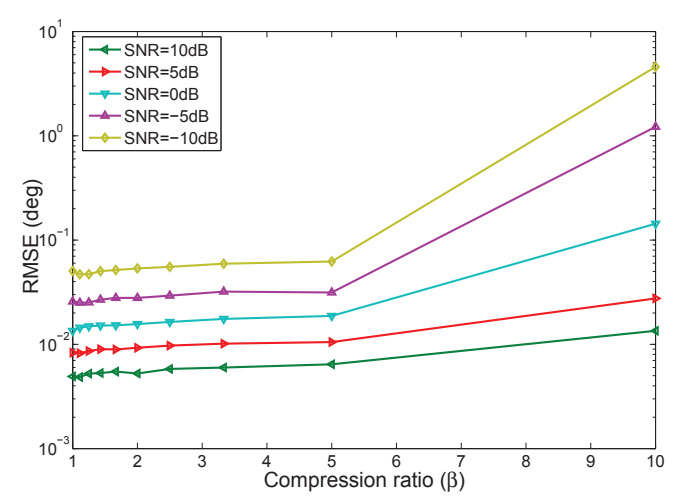

Fig. 3. The RMSE versus compression ratio.

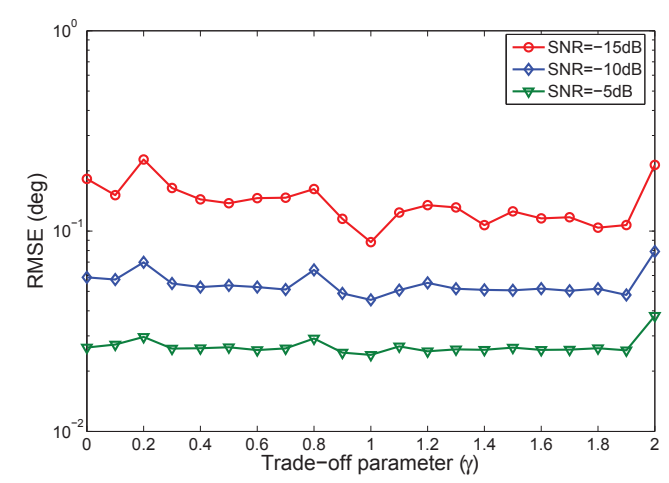

Fig. 4. The trade-off parameter $\gamma$ in low SNR.

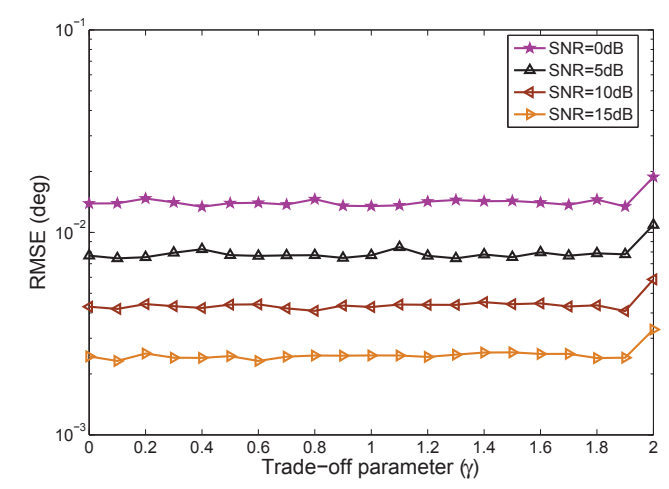

Fig. 5. The trade-off parameter $\gamma$ in high SNR. 
From Fig. 6, we can see that the proposed scheme and the LZYCB can provide higher resolution than other compressed schemes in the white noise. Furthermore, Figure 7 shows that the proposed scheme can also maintain high resolution in Gaussian colored noise. To illustrate the superiority of proposed scheme clearly, the RMSE of different schemes versus target numbers with $\mathrm{SNR}=10 \mathrm{~dB}$ is shown in Fig. 8 . In each case, 1,000 Monte-Carlo are carried out.

From Fig. 8, we can see that the proposed scheme can provide a higher precision than the LZYCB algorithm and Elad's algorithm. Moreover, the performance of proposed scheme can even surpass original high-dimension measurement under the fewer targets condition.

In the last simulation, we compare the DOA accuracy of different schemes versus SNR. Assume that there are 3 signals impinging from directions $\theta=\left[-3.15^{\circ}, 0^{\circ}, 3.15^{\circ}\right]$ in this simulation. The RMSE versus SNR under white noise and color noise are shown in Fig. 9 and Fig. 10, respectively. For each case, 1,000 Monte-Carlo are carried out.

From the Fig. 9, we can know that the proposed scheme has better estimation performance than other compressed schemes at low SNR. Note that the proposed scheme can reduce the mutual coherence between dictionaries, which result in a better performance than original high-dimension measurement in the high SNR. In Fig. 10, it is also proved that the proposed scheme has the ability to suppress the environment noise and provide well performance.

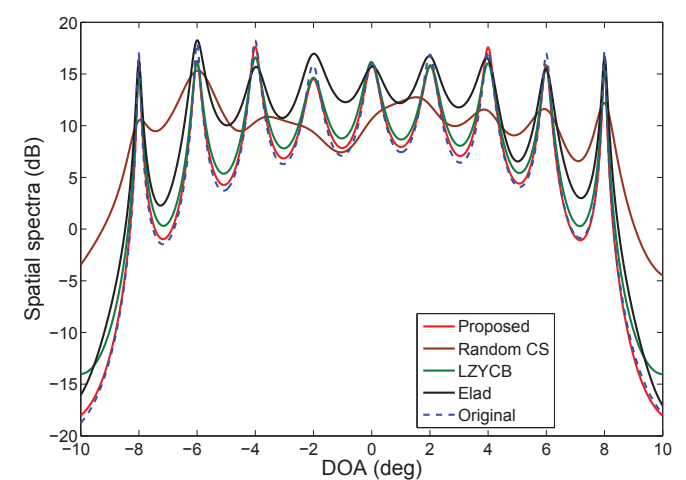

Fig. 6. Spatial spectra of DOA estimation under white noise.

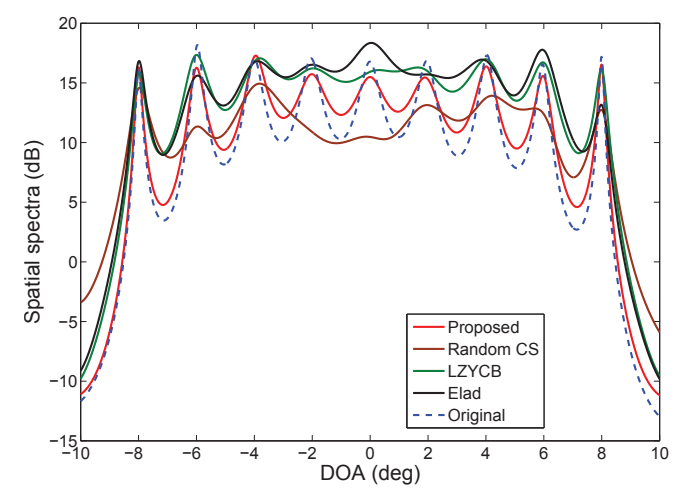

Fig. 7. Spatial spectra of DOA estimation under color noise.

\section{Conclusions}

In this paper, a robust measurement scheme is proposed to reduce computational complexity and suppress noise for DOA estimation in a Massive MIMO system. In this scheme, the optimization function is established by minimizing the mutual coherence of spatial sparse dictionary and the energy of measurement matrix. To solve this non-convex optimization problem, an alternating minimization scheme based on matrix factorization and Principal Component Analysis (PCA) is proposed. The specific optimization steps and the structure of measurement matrix are proposed. Simulation results prove the superiority of proposed scheme.

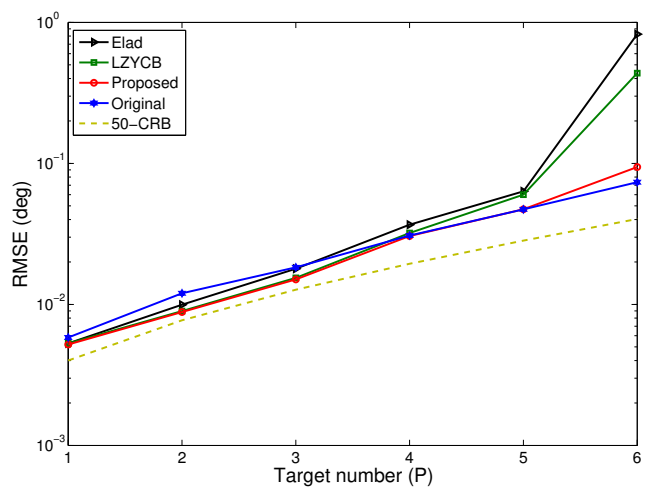

Fig. 8. The RMSE versus target number.

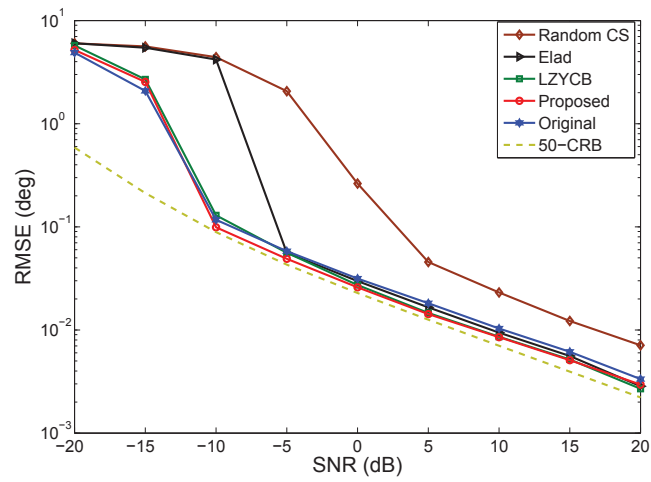

Fig. 9. The RMSE versus SNR under white noise.

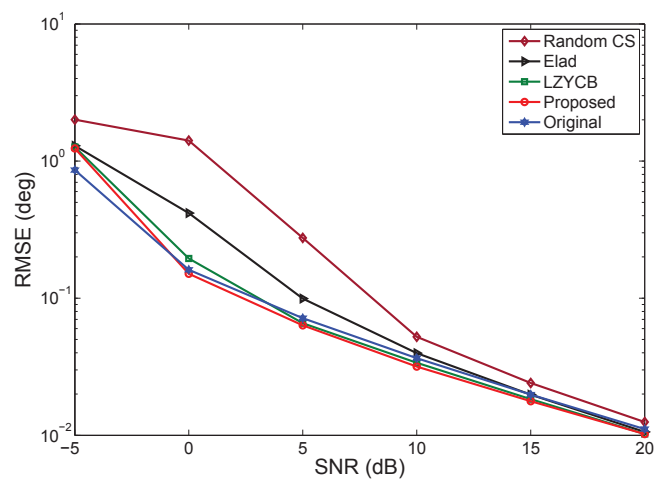

Fig. 10. The RMSE versus SNR under color noise. 


\section{Acknowledgments}

This work is supported by the National Nature Science Foundation (No. 61571148, No. 61871143), the Fundamental Research Funds for the Central Universities (No. HEUCFG201823) and the Fundamental Research Funds for the Central Universities (No. HEUCFP201836).

\section{References}

[1] KRIM, H., VIBERG, M. Two decades of array signal processing research: the parametric approach. IEEE Signal Processing Magazine, 1996, vol. 13, p. 67-94. DOI: 10.1109/79.526899

[2] LU, L., LI, G. Y., SWINDLEHURST, A. L., et al. An overview of massive MIMO: Benefits and challenges. IEEE Journal on Selected Topics in Signal Processing, 2014, vol. 8, no. 5, p. 742-758. DOI: 10.1109/JSTSP.2014.2317671

[3] JIANG, F., CHEN, J., SWINDLEHURST, A. L., et al. Massive MIMO for wireless sensing with a coherent multiple access channel. IEEE Transactions on Signal Processing, 2015, vol. 63, no. 12, p. 3005-3017. DOI: 10.1109/TSP.2015.2417508

[4] LARSSON, E. G., EDFORS, O., TUFVESSON, F., et al. Massive MIMO for next generation wireless systems. IEEE Communications Magazine, 2014, vol. 52, no. 2, p. 186-195. DOI: 10.1109/MCOM.2014.6736761

[5] HOCTOR, R. T., KASSAM, S. A. The unifying role of the coarray in aperture synthesis for coherent and incoherent imaging. Proceedings of the IEEE, 1990 , vol. 78, no. 4, p. 735-752. DOI: 10.1109/5.54811

[6] MOFFET, A. Minimum-redundancy linear arrays. IEEE Transactions on Antennas and Propagation, 1968, vol. 16, no. 2, p. 172-175. DOI: $10.1109 /$ TAP.1968.1139138

[7] BLOOM, G. S., GOLOMB, S. W. Applications of Numbered Undirected Graphs. Proceedings of the IEEE, 1977, vol. 65, no. 4, p. 562-570. DOI: 10.1109/PROC.1977.10517

[8] PAL, P., VAIDYANATHAN, P. P. Nested arrays: A novel approach to array processing with enhanced degrees of freedom. IEEE Transactions on Signal Processing, 2010, vol. 58, no. 8, p. 4167-4181. DOI: 10.1109/TSP.2010.2049264

[9] AHMED, A., ZHANG, Y. D., HIMED, B. Effective nested array design for fourth-order cumulant-based DOA estimation. In Proceedings of the IEEE Radar Conference (RadarConf). Seattle (USA), 2017, p. 998-1002. DOI: 10.1109/RADAR.2017.7944349

[10] VAIDYANATHAN, P. P., PAL, P. Sparse sensing with co-prime samplers and arrays. IEEE Transactions on Signal Processing, 2010 vol. 59, no. 2, p. 1405-1409. DOI: 10.1109/TSP.2010.2089682

[11] ROEMER, F., IBRAHIM, M., FRANKE, N., et al. Measurement matrix design for compressed sensing based time delay estimation. In Proceedings of the European Signal Processing Conference (EUSIP). Budapest (Hungary), 2016, p. 458-462. DOI: 10.1109/EUSIPCO.2016.7760290

[12] DONOHO, D. L. Compressed sensing. IEEE Transactions on Information Theory, 2006, vol. 52, no. 4, p. 1289-1306. DOI: $10.1109 /$ TIT.2006.871582

[13] WANG, Y., LEUS, G., PANDHARIPANDE, A. Direction estimation using compressive sampling array processing. In IEEE/SP Workshop on Statistical Signal Processing. Cardiff (UK), 2009, p. 626-629. DOI: 10.1109/SSP.2009.5278497

[14] ELAD, M. Optimized projections for compressed sensing. IEEE Transactions on Signal Processing, 2007, vol. 55, no. 12, p. 5695-5702. DOI: 10.1109/TSP.2007.900760
[15] CHEN, W., RODRIGUES, M. R., WASSELL, I. J. On the use of unit-norm tight frames to improve the average mse performance in compressive sensing applications. IEEE Signal Processing Letters, 2012, vol. 19, no. 1, p. 8-11. DOI: 10.1109/LSP.2011.2173675

[16] DUARTE-CARVAJALINO, J. M., SAPIRO, G. Learning to sense sparse signals: Simultaneous sensing matrix and sparsifying dictionary optimization. IEEE Transactions on Image Processing, 2009, vol. 18 , no. 7 , p. 1395-1408. DOI: 10.1109/TIP.2009.2022459

[17] ENTEZARI, R., RASHIDI, A. Measurement matrix optimization based on incoherent unit norm tight frame. AEU-International Journal of Electronics and Communications, 2017, vol. 82, p. 321-326. DOI: 10.1016/J.AEUE.2017.09.015

[18] LI, G., ZHU, Z., YANG, D., et al. On projection matrix optimization for compressive sensing systems. IEEE Transactions on Signal Processing, 2013, vol. 61, no. 11, p. 2887-2898. DOI: 10.1109/TSP.2013.2253776

[19] IBRAHIM, M., RAMIREDDY, V., LAVRENKO, A., et al. Design and analysis of compressive antenna arrays for direction of arrival estimation. Signal Processing, 2016, vol. 138, p. 1-14. DOI: 10.1016/j.sigpro.2017.03.013

[20] GU, Y., ZHANG, Y. D., GOODMAN, N. A. Optimized compressive sensing-based direction-of-arrival estimation in massive MIMO In Proceedings of the IEEE International Conference on Acoustics, Speech and Signal Processing-Proceedings (ICASSP). New Orleans (USA), 2017, p. 3181-3185. DOI: 10.1109/ICASSP.2017.7952743

[21] HONG, T., ZHU, Z. An efficient method for robust projection matrix design. Signal Processing, 2018, vol. 143, p. 200-210. DOI: 10.1016/j.sigpro.2017.09.007.

[22] TROPP, J. A., DHILlON, I. S., HEARTH, R. W., et al. Constructing packings in Grassmannian manifolds via alternating projection. Experimental Mathematics, 2008, vol. 17, no. 1, p. 9-35. DOI: $10.1080 / 10586458.2008 .10129018$

[23] TROPP, J. A., DHILlON, I. S., HEARTH, R. W., et al. Designing structured tight frames via an alternating projection method. IEEE Transactions on Information Theory, 2005, vol. 51, no. 1, p. 188-209. DOI: 10.1109/TIT.2004.839492.

\section{About the Authors ...}

Zhikai HUANG was born in 1992. He received his Bachelor Degree from Harbin Engineering University (HEU) in 2015, Heilongjiang, China. He's currently working on his $\mathrm{Ph}$.D Degree for Technology of Wireless Guidance in Department of Automation, Harbin Engineering University (HEU), Heilongjiang, China. His research interests in array signal processing and MIMO radar signal processing.

Wei WANG (corresponding author) was born in 1979. He received Ph.D. in Navigation, Guidance and Control from Harbin Engineering University (HEU), Heilongjiang, China, 2005. He was a Postdoctoral Research Associate at Harbin Institute of Technology, China (July 2006 to April 2009), Associate Professor at Harbin Engineering University (August 2008 to August 2010) and Academic Visitor at Loughborough University, UK (January 2010 to December 2010). He has published about 80 refereed journal and conference papers. He is a senior member of the Institute of Electrical and Electronic Engineers (IEEE). His current research interests include signal processing for wireless navigation systems and MIMO radar. 